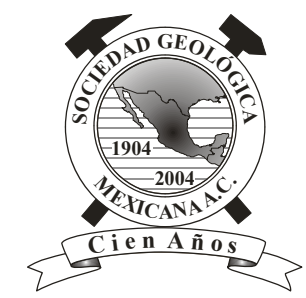

\title{
Estudio de flujos granulares de tipo geológico por medio del simulador multisensor GRANFLOW-SIM
}

\author{
Roberto Bartali ${ }^{1}$, Damiano Sarocchi ${ }^{2, *}$, Yuri Nahmad-Molinari ${ }^{3}$, \\ Luis Angel Rodríguez-Sedano ${ }^{4}$
}
${ }^{1}$ Doctorado Institucional en Ingeniería y Ciencias de Materiales, Instituto de Física, Universidad Autónoma de San Luis Potosí, Av. Dr. Manuel Nava 5, C.P. 78240 San Luis Potosí, México
${ }^{2}$ Instituto de Geología / DES Ingeniería, Universidad Autónoma de San Luis Potosí, Av. Dr. Manuel Nava 5, C.P. 78240 San Luis Potosí, México,
${ }^{3}$ Instituto de Física, Universidad Autónoma de San Luis Potosí, Av. Dr. Manuel Nava 5, C.P. 78240 San Luis Potosí, México, yuri@, dec1.ifisica.uaslp.mx
${ }^{4}$ Posgrado en Ciencias de la Tierra, Centro de Geociencias, Universidad Nacional Autónoma de México, Campus Juriquilla, 76230 Querétaro, México, 1rodriguez2021@live.com.mx.

*damiano.sarocchi@uaslp.mx

\begin{abstract}
Resumen
Las avalanchas y los flujos de escombro producidos por eventos catastróficos como las erupciones volcánicas se pueden entender como enormes flujos granulares. Debido a la imposibilidad de predicción y de la peligrosidad de dichos eventos por las altas temperaturas y las masas en movimiento involucradas, su estudio se limita a la observación remota y a la medición de los depósitos generados. La observación de estos fenómenos desde su inicio, es casi imposible de realizar dado que los tiempos de inicio y desarrollo son extremadamente cortos e imposibles de predecir con exactitud, además, debe llevarse a cabo desde una distancia considerable. El estudio del material depositado, ofrece importantes datos, pero sigue siendo un método indirecto para poder inferir la dinámica del flujo de escombros. En este artículo se describen las características del aparato GRANFLOW-SIM (Granular Flows Simulator), de sus sensores y el tipo de estudios que permite realizar. GRANFLOW-SIM es el primer aparato experimental en su tipo desarrollado y construido en México para el estudio y la simulación de diferentes tipos de flujos granulares observados en la naturaleza (tales como avalanchas, lahares y flujos de escombros) a escala y en tiempo real, por lo que emplea gran cantidad de sensores y cámaras de video de alta velocidad, repartidos a lo largo de las diferentes secciones que lo componen. La comprensión de los eventos precursores y detonadores, así como la manera en que estos flujos se desarrollan a lo largo de las pendientes volcánicas, es de fundamental importancia para prevenir desastres relacionados con ellos, salvaguardando así vidas e infraestructura. Una de las maneras en que estos fenómenos pueden ser estudiados y modelados es a través de aparatos experimentales que sean capaces de reproducirlos a escala. Estos aparatos experimentales deben permitir la medición del mayor número posible de variables involucradas durante el desarrollo del flujo, reproduciendo las características de laderas, barrancas naturales, condiciones de sedimentación y hasta la rugosidad del terreno. Puesto que el material que conforma la avalancha, se desplaza a gran velocidad, la medición de sus características en tiempo real implica un reto tecnológico, tanto en eventos observados en el campo como en los simuladores, requiriendo mucha velocidad para la captura de los datos y gran capacidad de almacenamiento de la información.
\end{abstract}

Palabras clave: experimentos a gran escala, flujos granulares, avalanchas, flujos de escombros, flujos piroclásticos.

\author{
Abstract \\ Avalanches and debris flows caused by catastrophic events such as volcanic eruptions can be seen as huge granular flows. Due to
}


the impossibility of prediction and danger of such events, given the high temperatures and the moving masses involved, their study is limited to remote observation and measurement of the deposits generated. The observation of these phenomena from the beginning is almost impossible because the start and development are extremely short and impossible to predict accurately; furthermore, it has to be done from a considerable distance. The study of deposited material provides important information, but it is still an indirect method to infer the dynamics of a debris flow. This article describes the characteristics of the device GRANFLOW-SIM (Granular Flows Simulator), its sensors, and the type of studies that it is able to do. GRANFLOW-SIM is the first experimental unit of its kind designed and built in Mexico to study and simulate different types of granular flows observed in nature (such as landslides, lahars, and debris flows) at real scale and in real-time, employing a large number of sensors and high speed video cameras spread throughout the different sections that comprise it. The understanding of the precursor events and trigger mechanisms, as well as how these flows develop along the volcanic slopes, are of fundamental importance in preventing disasters associated with them, and therefore safeguarding lives and infrastructure. One way in which these phenomena can be studied and modeled is through an experimental apparatus capable of reproducing them in scale. These experimental apparatuses should allow measurement of the maximum number of variables involved during the development of a flow, reproducing the features of hillsides, natural ravines, sedimentation conditions and roughness of the terrain. Since the material that constitutes the avalanche travels at high speed, the measurement of its characteristics in real time implies a technological challenge in both events observed in the field and in the simulators, requiring high speed data collection and large storage information capacity.

Keywords: large scale experiments, granular flows, debris avalanches, debris flows, pyroclastic flows.

\section{Introducción}

Un material granular está compuesto por una gran cantidad de partículas (por ejemplo grava, arena, maíz) de diferente tamaño, densidad y forma que se pueden mover independientemente e interactuar entre ellas (Kanadoff, 1999). Con esto en mente, los flujos granulares se pueden definir como el movimiento y la interacción de grandes cantidades de partículas y los podemos encontrar en muchos procesos industriales, agrícolas y de la vida cotidiana.

El comportamiento del material granular es muy peculiar, presentando fases en las que se asemeja a un fluido, otras en las que actúa como un gas y, la mayor parte de las veces, una compleja combinación de ambos (Jaeger et al., 1996). Esto es debido a la manera en que se transfiere colisionalmente la energía entre las partículas y a la manera en que la energía total del conjunto se disipa (Drake, 1990; Ippolito et al., 1995). La interacción puede depender de variables como el diámetro, la forma, la pendiente del plano de deslizamiento, la geometría del confinamiento, la densidad, resistencia y dureza del material, la cantidad de lubricantes presentes al inicio, durante y al final del flujo y del tipo de evento detonador.

En la naturaleza podemos encontrar ejemplos de estos: flujos de escombros, avalanchas de escombros, flujos piroclásticos, avalanchas de nieve y flujos generadores de dunas, entre otros. En el caso de las avalanchas, flujos de escombros y flujos piroclásticos, las partículas son rocas (clastos) cuyas dimensiones abarcan varios órdenes de magnitud, desde pocas micras en la matriz, hasta decenas de metros de diámetro para los bloques de mayor dimensión. Los flujos granulares son extremadamente complejos de entender y modelar por la gran cantidad de variables en juego, el breve tiempo en el cual se inician y desarrollan y el carácter multi-fase del material involucrado.
La comprensión de un fenómeno natural, depende en gran medida de la posibilidad de observarlo mientras sucede, de poder realizar la medición de las variables involucradas, y de la capacidad de modelar y de experimentar con éstas en un ambiente controlado. Así, el estudio de la fenomenología relacionada con las avalanchas granulares se puede llevar a cabo de diferentes maneras: 1) por medio de la observación directa, 2) el análisis de los depósitos, 3) la creación de modelos teóricos, y 4) la simulación a escala con experimentación en laboratorio. Solo a través de la aplicación de cada metodología y su interrelación, se puede obtener una visión global del fenómeno para lograr su comprensión integral.

\subsection{Observación directa del fenómeno}

El momento exacto en el que se desarrolla una avalancha, un flujo piroclástico o un flujo de escombros, en la mayoría de los casos, no puede ser pronosticado con exactitud. Son pocos los ejemplos de observaciones directas y desde un lugar cercano, debido a la peligrosidad extrema del fenómeno (Sulpizio et al., 2010). Aun considerando dispositivos remotos autónomos y diseñados para soportar condiciones extremas de temperatura, presión y vibraciones, la duración del flujo es muy breve y la nube de ceniza que se desarrolla por el movimiento y la fracturación progresiva de la masa de rocas, dificulta la observación directa de lo que sucede en su interior (Sarocchi, 2007).

\subsection{Análisis de los depósitos}

La manera más segura de obtener datos acerca de los flujos granulares es por medio del estudio de las texturas, las estructuras, y la morfología de los depósitos. Éste se realiza por medio de la descripción detallada en el campo, 
la recolección y el análisis de muestras. La comparación de sus características previas al evento y posteriores, proporciona sin duda datos valiosos. Sin embargo, flujos granulares de gran magnitud son capaces de modificar las características del medio circundante y acarrear materiales que se encontraban in situ a lo largo de su recorrido. Durante el tiempo que transcurre antes de que se pueda estudiar un depósito, la erosión debida a la lluvia, al viento, o a la nieve, ayuda a la removilización del material que lo compone, dificultando la interpretación real de lo sucedido.

\subsection{Modelos teóricos}

La escasez de datos observacionales, el desconocimiento parcial de las condiciones iniciales y sobre todo la imposibilidad de medir las condiciones internas de un volcán o del material que se movilizará en una avalancha, dificultan la creación de modelos teóricos capaces de explicar completamente los fenómenos. Hasta la fecha no existe algún método capaz de predecir el momento exacto en el cual se desarrollará una erupción o una avalancha, tampoco se tiene suficiente información para poder evaluar el alcance o el grado de peligrosidad real que esta tendría, sino que solo en base a eventos registrados en el pasado (Iverson et al., 1998) es como se pueden inferir estos parámetros.

La interacción con el medio ambiente, por lo general, modifica el desarrollo del flujo y la sedimentación del material, por lo que la predicción de estos eventos y su desarrollo son muy complejos. Así, crear modelos teóricos con tantas variables involucradas resulta muy complejo, aun conociendo la historia eruptiva de un volcán.

\subsection{Experimentación en laboratorio}

Cuando se trata de simular flujos granulares relacionados con actividad volcánica (flujos de bloques y ceniza, avalanchas de escombros, flujos piroclásticos), uno de los problemas más recurrentes es que el material original, se encuentra en condiciones que difícilmente se pueden reproducir y manejar en un laboratorio. La escala es tan reducida que la interacción entre los materiales no refleja del todo la realidad (Cagnoli y Romano, 2010). En muchos casos se utilizan materiales artificiales cuyo comportamiento sea semejante al del material natural. La ventaja de esto es que el experimento se lleva a cabo en condiciones de varios órdenes de magnitud inferiores a las reales, y los experimentos se pueden repetir una gran cantidad de veces. Sin embargo, los resultados obtenidos en experimentos a pequeña escala, resultan ser solo una aproximación a la realidad porque las energías en juego son mucho menores y no se toman en cuenta otros factores como las altas temperaturas o la resistencia del material que fluye, de ahí la dificultad en escalar por completo experimentos de este tipo.

Una manera de resolver parcialmente ésta problemática es la de construir equipos experimentales de gran escala por medio de los cuales sea posible reproducir, más fielmente, las condiciones naturales y medir, en un ambiente controlado, las variables asociadas al comportamiento del material. Los resultados obtenidos en estas condiciones permiten crear bases de datos que facilitan la comprensión de los fenómenos y la creación de modelos teóricos. La comparación de los resultados obtenidos, con los datos proporcionados trabajando con materiales artificiales es de gran utilidad para determinar las ecuaciones que modelan los fenómenos naturales. La experimentación a escala sirve también para desarrollar y probar los sensores y equipos de medición que pueden ser utilizados en el campo. El desarrollo y la fabricación de estos aparatos experimentales, es un trabajo multidisciplinario en el cual la conjunción de conocimientos geológicos, electrónicos, computacionales, físicos y de sistemas de comunicación, es fundamental para una más eficaz comprensión, interpretación y aplicación de los resultados.

\section{Avalanchas, flujos de escombros y flujos piroclásticos}

Las avalanchas son flujos enormes y muy rápidos generados a partir del colapso gravitacional de sectores de edificios volcánicos y no volcánicos, son fenómenos altamente catastróficos que han sido estudiados por numerosos autores (Gorshkov, 1959; Hsü, 1975; Voight et al., 1981; Siebert, 1984; Palmer et al., 1991; Capra et al., 2002; Cortés et al., 2010). Los flujos de escombros son una mezcla de sedimentos pobremente clasificados y agua que se mueven bajo el efectos de la gravedad (Iverson, 1997), se presentan en una gran variedad de ambientes (Sharp y Nobles, 1953; Curry, 1966; Pierson, 1980; Innes, 1983; Van Stejin et al., 1988; Hubert y Filipov, 1989; Nieuwenhizen y Van Stejin, 1990; Harris y Gustafson, 1993; Rickenmann y Zimmermann, 1993; DeGraff, 1994; Boeolhouwers et al., 2000; Pareschi et al., 2000; Rodolfo, 2000). Cuando el material removilizado es de origen volcánico el flujo de escombros se denomina lahar (Iverson, 1997; Vallance, 2000). Los flujos piroclásticos son una mezcla de gas y partículas que pueden deslizarse a lo largo del terreno con velocidades de más de $250 \mathrm{~km} / \mathrm{h}$, y temperaturas mayores a $\operatorname{los} 500^{\circ} \mathrm{C}$. Pueden ser originados por destrucción de domos o colapsos de columnas eruptivas (Carey, 1991; Sato et al., 1992; Druit, 1998; Branney y Kokelaar, 2002; Sulpizio y Dellino, 2008). Estos tres fenómenos son flujos granulares que se desarrollan en medios secos, húmedos o gaseosos, en laderas con una pendiente no necesariamente superior al ángulo de reposo del material, en ambientes confinados como barrancas y valles o en lugares completamente abiertos. Normalmente la masa, que puede alcanzar magnitudes del orden de $10^{5}-10^{6} \mathrm{~m}^{3}$, se desliza a gran velocidad y se detiene a distancias de hasta decenas de $\mathrm{km}$ del punto de origen. Se pueden originar antes, durante o después de una erupción volcánica, no necesariamente en las laderas del volcán. 
El tamaño del material granular que conforma esta clase de flujos comprende un amplio rango que abarca por lo menos 8 órdenes de magnitud. La textura, dureza, densidad, forma, composición mineralógica y temperatura del material involucrado es totalmente heterogénea. Su comportamiento y características son fácilmente modificados durante el flujo y después de su sedimentación, tanto por factores externos (climatológicos), como internos (mecánicos), debido a los impactos mutuos entre partículas y la fricción con el medio en el cual se desarrollan.

Básicamente los flujos piroclásticos, los flujos de escombro y las avalanchas son controlados por la gravedad, pero los mecanismos de disparo y de transporte son diferentes y la interacción con el medio determina cambios dinámicos. La energía cinética del flujo es disipada principalmente por la interacción entre las partículas y por la fricción basal, pero es tan grande que le permite, en ocasiones, superar barreras topográficas del orden de cientos de metros (Saucedo, 1997) y modificar radicalmente la topografía circundante.

\section{Descripción del aparato experimental}

GRANFLOW-SIM (Figura 1) es un aparato experimental modular diseñado para realizar experimentos y modelar avalanchas, flujos de escombros y flujos piroclásticos (secos y con presencia de agua). Es un sistema muy versátil cuyas características geométricas pueden ser modificadas rápidamente para adaptarse a las necesidades experimentales. Posee una gran variedad de sensores colocados estratégicamente que permiten medir, en tiempo real, las variables físicas que se quieren estudiar. Se compone de las siguientes secciones: 1) contenedor para el material granular; 2) unidad de aceleración; 3) canaleta con inclinación variable y 4) depósito para la sedimentación.

\subsection{Contenedor del material granular}

El contenedor del material granular es un recipiente con una capacidad de $0.014 \mathrm{~m}^{3}$ y puede contener aproximadamente $20 \mathrm{~kg}$ de material, dependiendo de la densidad y de las clases granulométricas de la mezcla utilizada. La capacidad del contenedor se puede incrementar agregando una o más secciones. El fondo del contenedor tiene dos puertas que se abren simultáneamente para permitir que el material caiga por gravedad sin interferencia alguna.

\subsection{Unidad de aceleración}

La unidad de aceleración es necesaria para proporcionar al flujo una velocidad inicial diferente de cero. El incremento de la velocidad se logra por medio de la caída vertical del material desde diferentes alturas. Esta sección está compuesta por dos tramos: uno vertical donde el material sufre una caída libre y uno curvo que une el tramo vertical

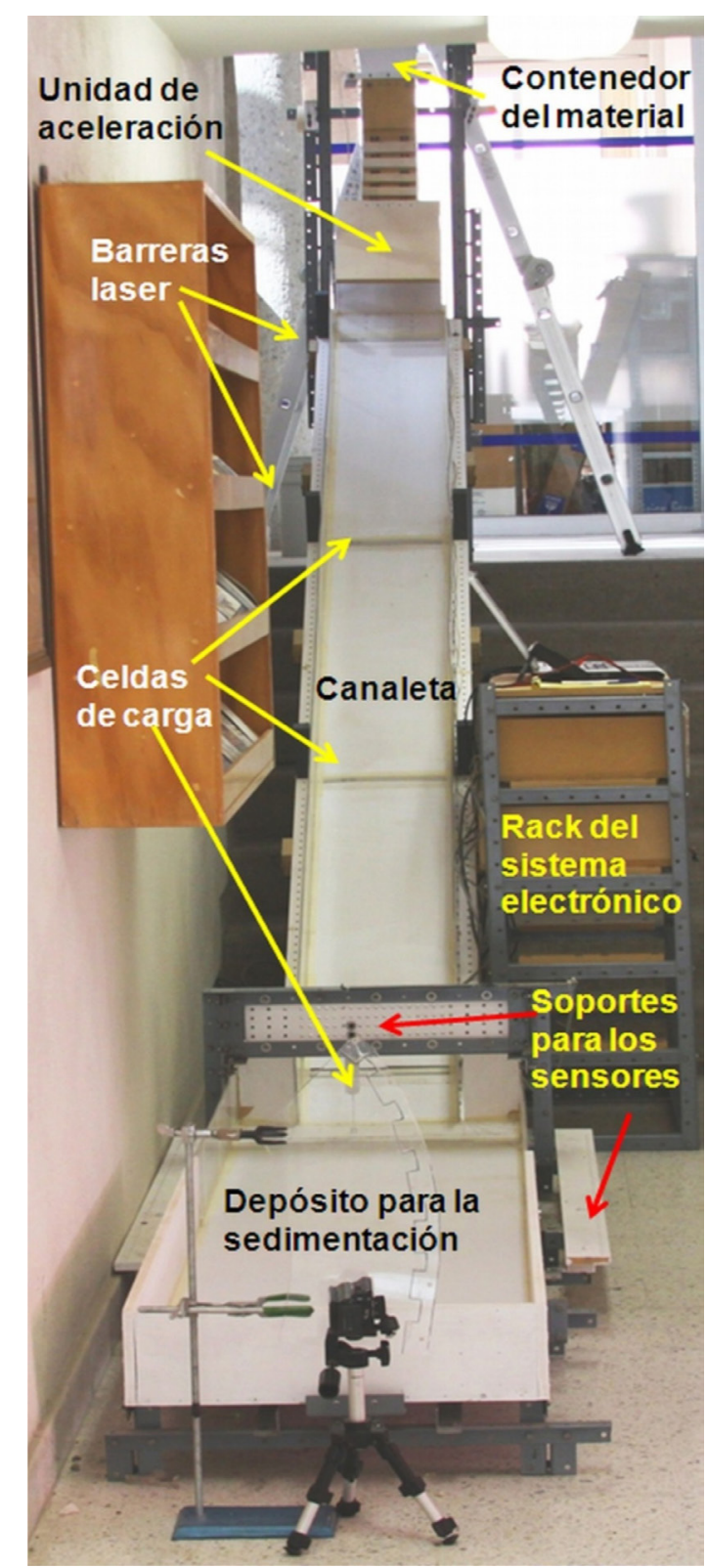

Figura 1. Diferentes partes que conforman el aparato GRANFLOW-SIM, se puede apreciar la modularidad del aparato.

con la canaleta inclinada reduciendo al mínimo el cambio de pendiente evitando que el flujo modifique sus características.

\subsection{Canaleta con inclinación variable}

La canaleta, tiene una longitud de $4.3 \mathrm{~m}$ y un ancho de $0.3 \mathrm{~m}$. Está fabricada de madera y las paredes son de acrílico transparente de $0.2 \mathrm{~m}$ de altura. La parte de madera es soportada por una estructura metálica modular que le da rigidez y resistencia manteniendo reducido el peso. Las paredes transparentes permiten adquirir fotografías y videos desde cualquier punto. La canaleta tiene soportes que pueden ser movidos libremente a lo largo de la rampa, para la 
colocación de los distintos tipos de sensores. La inclinación de la canaleta puede ser modificada en un rango entre $15^{\circ}$ y $70^{\circ}$. La longitud de la canaleta puede ser incrementada agregando uno o más módulos, la pendiente de cada uno de ellos puede ser distinta, o bien, se puede introducir un sector curvo con el objetivo de simular las barrancas naturales con la mayor fidelidad posible, además se pueden colocar superficies de diferente rugosidad.

\subsection{Unidad de sedimentación}

La unidad de sedimentación es la última sección del equipo y es donde el material desacelera y se deposita; normalmente este tramo se mantiene horizontal, pero puede ser inclinado en un rango de $0^{\circ}$ a $5^{\circ}$. A lo largo de esta sección se pueden colocar diversos tipos de sensores que, aunados a las cámaras de alta velocidad, permiten estudiar la forma en que el material desacelera, se detiene y se sedimenta, además de la fuerza de impacto que ejerce el flujo, proporcionando información importante acerca de la potencia asociada a la avalancha, lo cual permite inferir acerca de los riesgos relacionados con eventos a escala real.

\section{Sistema de sensores}

La comprensión de un fenómeno natural, depende de la capacidad de observar y medir las variables que lo gobiernan, por esta razón es muy importante utilizar la mayor cantidad de sensores posible, que estos puedan tomar datos en tiempo real y que puedan ser colocados en diferentes partes del sistema.

Las escalas de tiempo en las que se desarrollan los flujos en la naturaleza van de minutos a horas, pero en la experimentación a escala, esto se reduce a pocos segundos. El sistema electrónico de control de GRANFLOW-SIM tiene la capacidad de leer y almacenar los datos proporcionados por cada uno de los sensores a una tasa de 1000 veces por segundo y con una resolución de 16 bits. Todo el sistema de amplificación, acondicionamiento de señal y control de cada uno de los sensores ha sido desarrollado específicamente con el fin, no solo de ser utilizado en este equipo experimental, sino de que constituya la base para construir equipos que puedan ser colocados en barrancas naturales para el estudio de fenómenos reales.

Los sensores utilizados son: 1) celdas de carga, 2) barreras laser, 3) cámaras fotográficas, 4) cámaras de video de alta velocidad, 5) sensores de presión de aire, 6) transductores piezoeléctricos, 7) micrófonos, 8) sensores de temperatura, 9) sensores de humedad y 10) antenas RFID (Radio Frequency Identification Device).

\subsection{Celdas de carga}

Son dispositivos que miden la presión que ejerce el material (en 5 puntos fijos distintos) mientras se desliza en la canaleta y en la unidad de sedimentación. Las celdas están colocadas horizontalmente, de tal manera que la presión ejercida por el flujo sea siempre perpendicular a la celda, para medir el peso real del material, independientemente de la inclinación de la canaleta. En la unidad de sedimentación se han colocado también dos celdas de carga verticalmente, dispuestas de frente al flujo, cuya posición y ubicación pueden ser modificadas, con las que se mide la fuerza y la presión debida al impacto del material.

\subsection{Barreras laser}

Con estas barreras laser se miden la velocidad, la aceleración, se reconstruye la topografía dinámica y su evolución a lo largo de la unidad de aceleración, de la canaleta y de la unidad de sedimentación. Están constituidas por parejas de barras que contienen por un lado una serie de láseres a $633 \mathrm{~nm}$ y por el otro lado un conjunto de fotodiodos. En cada barra se encuentran 8 láseres y 8 fotodiodos. En total se utilizan 8 barreras colocadas estratégicamente a lo largo del recorrido del flujo. La mínima distancia posible entre una barrera y la otra es de $3.5 \mathrm{~cm}$ y la resolución vertical es de $1.5 \mathrm{~cm}$. En la unidad de sedimentación se mide la altura del depósito y la desaceleración.

\subsection{Cámaras fotográficas}

El uso de cámaras fotográficas tiene varios objetivos: el registro y la documentación del desarrollo del flujo, de los micro-depósitos en las paredes de plástico transparente y de los depósitos consolidados y seccionados. Por medio de las fotografías de alta resolución se realizan estudios granulométricos ópticos a detalle (Sarocchi et al., 2005) y de la micro-fabrica (Capaccioni et al., 1997), también se analizan la distribución del material depositado, el ángulo de apertura del depósito y su alcance.

\subsection{Cámaras de video de alta velocidad}

Las cámaras rápidas son un instrumento muy importante cuando se trata de observar fenómenos que ocurren en tiempos muy breves. Un flujo en un simulador como GRANFLOW-SIM, puede alcanzar $60 \mathrm{~km} / \mathrm{h}$., los videos de alta velocidad permiten congelarlo para estudiar su desarrollo, determinar cómo se comporta cada clasto y como interactúa con los demás. El video, junto con la información de las celdas de carga, permite cuantificar la temperatura granular del flujo, construir su perfil de velocidad y su granulometría dinámica, con mucha resolución. La toma de videos desde la parte frontal y superior, permiten reconocer la formación de frentes, de ondas de densidad y de la influencia de obstáculos y paredes durante el desarrollo del flujo. 


\subsection{Sensores de presión de aire}

La masa del flujo en rápido movimiento produce diferencias de presión en el aire circundante las cuales modifican el comportamiento de parte del material (Roche et al., 2010). Las diferencias de presión que se generan en los alrededores de los flujos granulares pueden proporcionar información sobre su reología y la dispersión del material fino hacia la atmósfera (elutriación). La formación de ondas de choque es un fenómeno tan violento como el impacto de la avalancha misma, por lo que es muy importante comprender su formación y propagación.

\subsection{Transductores piezo-eléctricos}

El material en el interior del flujo durante el deslizamiento en la canaleta tiene un comportamiento caótico, generando gran cantidad de impactos entre las partículas, los cuales impulsan algunas y le restan energía a otras. Por medio de transductores piezoeléctricos, colocados en la parte inferior de la canaleta, se mide la cantidad de impactos y su intensidad, permitiendo calcular la temperatura granular del flujo. El comportamiento global del flujo (velocidad, alcance, segregación de partículas) depende de la energía disipada en las colisiones y de la fricción con la base de la canaleta.

\subsection{Micrófonos}

La función de los micrófonos es semejante a la de los transductores piezo eléctricos, con la diferencia de que estos se colocan en la parte superior de la canaleta. La correlación de la información generada por los transductores y por los micrófonos permite obtener datos más completos acerca de la interacción entre los clastos y también determinar cambios drásticos en el régimen del flujo (Drake, 1990; Yamasato, 1997) como por ejemplo la transición de un régimen puramente colisional a uno friccional o para distinguir entre un flujo laminar y uno turbulento.

\subsection{Sensores de temperatura}

Las colisiones entre las partículas que integran el flujo producen disipación de energía, parte de la cual es transformada en calor. No existen estudios de este tipo en experimentos a gran escala, pero es técnicamente posible detectar variaciones mínimas entre la temperatura inicial en el contenedor del material y la final en la unidad de sedimentación.

\subsection{Sensores de presión de poro y humedad}

La presión de poro es un dato muy importante para la comprensión de los mecanismos que disparan los flujos de escombros y determinan su elevada movilidad (Costa, 1988; Roche et al., 2010) y se mide por medio de un piezocono miniatura. La cantidad de líquidos influye en el comportamiento global del flujo y se modifica constantemente debido a la segregación y a la dispersión (evaporación, absorción, etc.). La cantidad de agua se mide primero en el contenedor del material y después en el depósito cuando el flujo se detiene.

\subsection{Antenas RFID (Radio Frequency Identification)}

Son unas pequeñas antenas colocadas en forma de anillo alrededor de la canaleta que se comunican con los transponders utilizados como partículas rastreadoras electrónicas. Las antenas proporcionan pulsos cada vez que detectan una de las partículas rastreadoras, independientemente de su posición en el interior del flujo, generando datos útiles para realizar estudios de velocimetría diferencial.

\section{Materiales utilizados}

Para que la experimentación proporcione datos significativos es necesario trabajar con materiales ideales y naturales para simular de una forma más realista lo que ocurre en los flujos granulares reales. En los experimentos se utilizan tres tipos de materiales (Figura 2): 1) naturales, 2) artificiales y 3) partículas rastreadoras.

Los materiales naturales se han recolectado en los volcanes Nevado de Toluca, Volcán de Colima y en la Joya Honda en San Luís Potosí. Todos han sido seleccionados y tamizados obteniendo fracciones pertenecientes a 9 clases granulométricas $(-4 \varphi$ a $4 \varphi)$; adicionalmente se utilizan varios tipos de arcillas (caolinita, bentonita) y pómez.

Los materiales artificiales son esferas de vidrio de diferente tamaño (10-20 $\mu \mathrm{m}$ a $20 \mathrm{~mm}$ ), para estudios sobre el efecto de la densidad se utilizan también esferas de acero y plástico.

Las partículas rastreadoras son materiales naturales y artificiales que se introducen, en pequeñas cantidades, en la masa del flujo, con la finalidad de poder determinar con precisión el comportamiento selectivo del flujo. Otro tipo de partícula rastreadora son los transponders (receptorestransmisores miniatura) que casi siempre se encuentran ocultos en el interior del flujo. Cuando se realizan experimentos con materiales de mayores dimensiones $(\geq$ $-4 \varphi$ ) y canaletas de mayor longitud y ancho, se utilizan giroscopios miniaturizados los cuales permiten medir en tiempo real, vía radio frecuencia, su comportamiento en el interior del flujo.

\section{Tipos de experimentos planeados}

El simulador GRANFLOW-SIM ha sido diseñado y construido para realizar una gran variedad de experimentos dirigidos a la comprensión de los flujos granulares en 

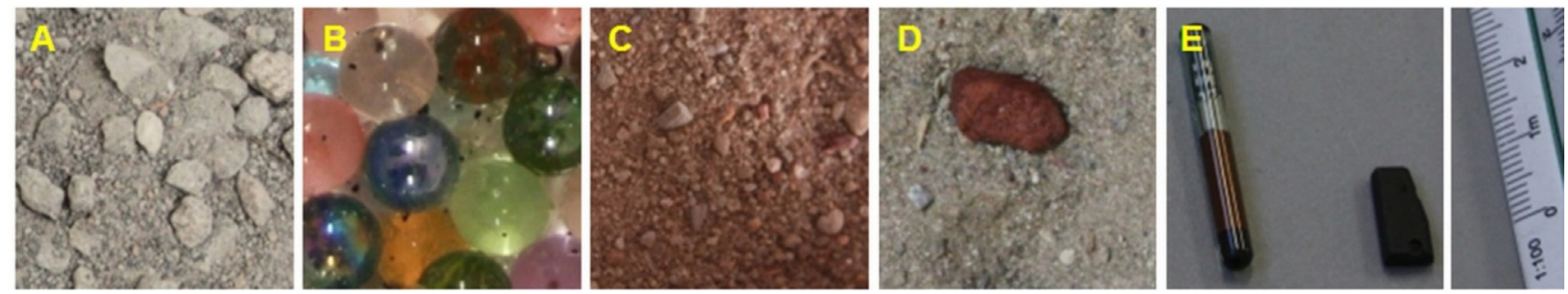

Figura 2. Materiales usados en los experimentos. Materiales naturales (A, C, D), materiales naturales coloreados artificialmente llamados partículas rastreadoras naturales $(\mathrm{C} \mathrm{y} \mathrm{D)}$, transponders (E) y materiales esféricos artificiales como canicas, esferas de hidrogel y balines de acero (B).

general, y en particular a los movimientos de masa de tipo geológico. Se trata de un instrumento muy versátil debido a su modularidad mecánica y los diferentes sensores con que cuenta. Estos sensores pueden ser colocados en diferentes posiciones y ubicaciones dependiendo del tipo de experimento que se está realizando.

Los experimentos para los cuales se ha creado el equipo están dirigidos al estudio de: 1) La cinemática y la reología del flujo (velocidad, aceleración, alcance, temperatura granular) en función de las siguientes variables: masa inicial, granulometría, velocidad inicial, inclinación de la canaleta, presencia de lubricantes (arcilla, limo, agua) o rugosidad del fondo. 2) Los procesos dinámicos que ocurren durante el desarrollo del flujo (mecanismos de transferencia de la cantidad de movimiento entre clastos, interacción con el fondo, transformación entre régimen colisional a friccional, mecanismos de segregación durante el transporte, formación de frentes secundarios u ondas de densidad, formación y evolución de carpetas de tracción, estructuras dinámicas internas, gradientes de velocidad, leveés, desacoplamiento entre avalancha basal y nube de material fino, generación y propagación de ondas de choque). 3) Los mecanismos de desaceleración y detención del flujo (geometría del depósito, formación de gradaciones granulométricas verticales y laterales, desarrollo de una fábrica en las partículas, grado de compactación). 4) Los umbrales que determinan un cambio de régimen reológico (cantidad de arcilla, limo, agua, granulometría, densidad y forma). 5) Los procesos de erosión, incorporación de material (bulking), depósito y sus relaciones con la reología del flujo, la geometría de la canaleta y la rugosidad del fondo. 6) Los procesos de diferenciación que se instauran en el flujo (segregación por densidad, forma y tamaño de los clastos).

\subsection{Resultados preliminares}

GRANFLOW-SIM ofrece la posibilidad de realizar una amplia gama de experimentos. Así, al verificar su funcionalidad y comprobar el correcto funcionamiento de los sensores se obtuvieron los siguientes resultados preliminares.

\subsection{Segregación y formación de ondas de densidad}

La masa de material que se desliza a lo largo de la canaleta sufre segregación y se forman ondas de densidad que son visibles a simple vista en las imágenes tomadas con ayuda de la cámara de alta resolución temporal (Figura 3C). Las mediciones en tiempo real realizadas por medio de las celdas de carga evidencian claramente la formación de estas perturbaciones y aún más, permiten medir la intensidad de las presiones generadas sobre las celdas. Las graficas de la Figura $3 \mathrm{~A}$ se refieren a la señal detectada por tres celdas de carga en un experimento realizado utilizando una avalancha granular seca (masa de $22.7 \mathrm{Kg}$ ) de partículas naturales polidispersas distribuidas según una función de Weibull (Figura 3B). Las celdas de carga se encuentran colocadas al inicio, a la mitad y al final de la canaleta. Se puede observar que, inmediatamente después de que la avalancha empieza a desplazarse por la canaleta, se forma un primer frente el cual se propaga y genera a su vez otros más. Un hecho interesante es que el mismo patrón ha sido documentado en la gran mayoría de los experimentos realizados lo que comprueba en primer lugar la reproducibilidad de los experimentos y que la formación de ondas de densidad es un fenómeno muy común en los flujos granulares (Hoblitt, 1986; Hupper et al., 1986).

\subsection{Velocidad diferencial en el cuerpo del flujo}

La toma de videos de alta velocidad (270 y $1000 \mathrm{fps}$ ) por medio de cámaras rápidas ha permitido observar el movimiento del material durante el deslizamiento del flujo (Figura 3C) y congelar imágenes donde es evidente la presencia de una gradación de velocidad con desarrollo de superficies críticas donde la velocidad varía drásticamente en pocos milímetros de espesor. También es posible observar y medir el espesor de las dos capas y su evolución a lo largo del proceso que dura no más de un segundo. En este muy breve lapso de tiempo, por medio de las cámaras utilizadas se pueden tomar cientos y hasta mil fotogramas que permiten observar el fenómeno en cámara lenta. A partir de estas imágenes, por medio de un software especializado (Martínez-Ramírez y González, 2006) o de forma semi- 

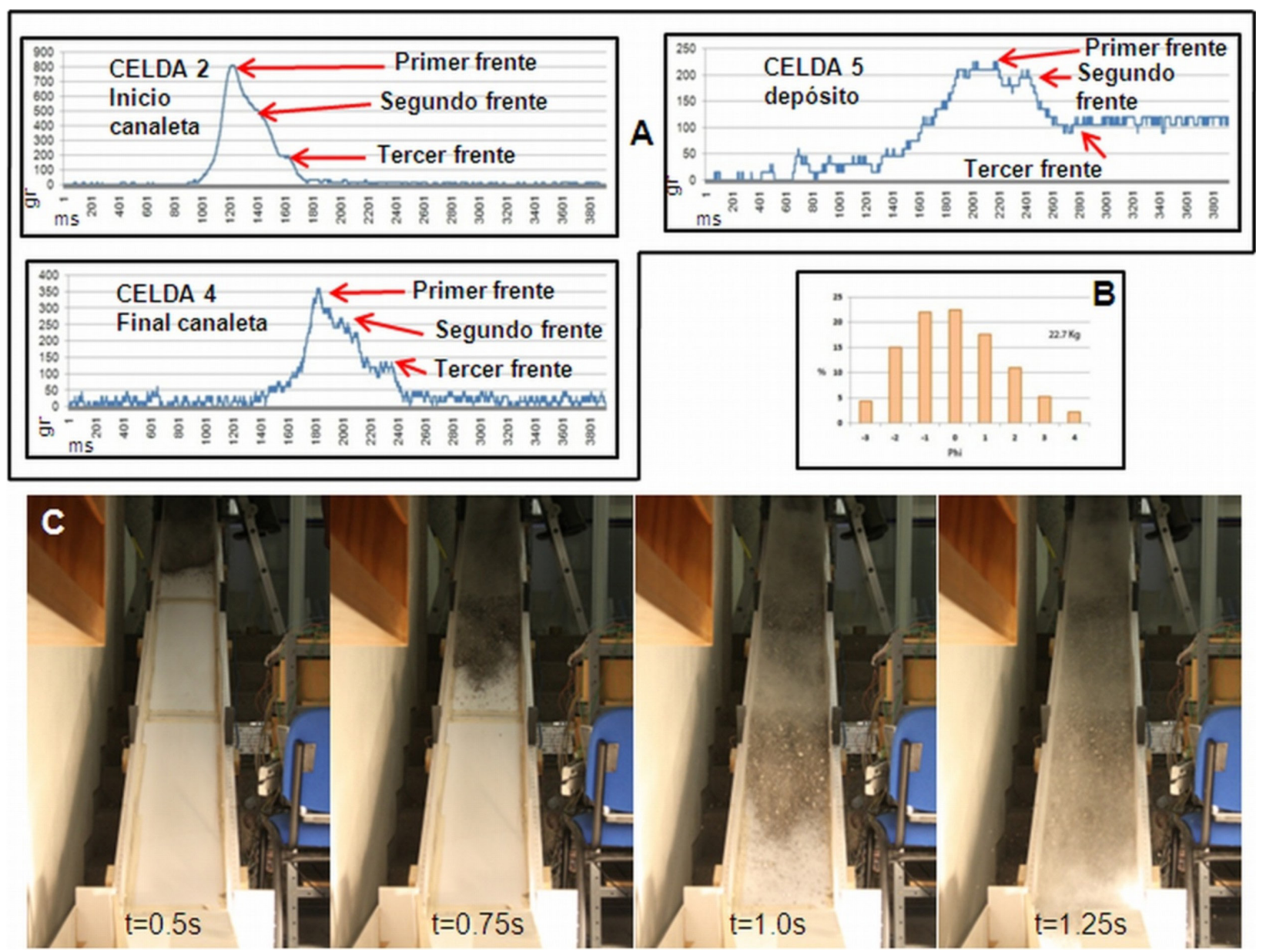

Figura 3. Ejemplos de gráficas construidas a partir de datos obtenidos por las diferentes celdas de carga (A). Se aprecia cómo la segregación del material, observada en las fotografías (C), corresponde con tres cambios bien definidos en dichas gráficas evidenciados con las flechas de color rojo. Se muestra la distribución granulométrica del material lanzado (B).

manual, se realizan estudios de velocimetría cuantitativa (Figura 4) por medio de los cuales se pueden medir: 1) velocidades diferenciales entre capas, 2) espesores de las diferentes capas con diferente velocidad o formación de bandas por esfuerzo cortante, 3 ) velocidad de los clastos en función de su tamaño, 4) trayectorias de las partículas e incluso el momento rotacional de los clastos mayores.

\subsection{Formación de gradaciones en los depósitos}

El proceso de agregación en su estadio terminal, fracciones de segundos antes del congelamiento del flujo, fue analizado, fotograma por fotograma, por medio de las cámaras de alta velocidad. Las imágenes han sido procesadas y a cada una se aplicaron métodos de granulometría óptica cuantitativa basados en el análisis de Rosiwal (Sarocchi et al., 2005). Estos estudios han permitido construir los perfiles granulométricos verticales del flujo y de los depósitos (Figura 5), en los cuales se aprecia la típica gradación inversa que se observa en muchos depósitos piroclásticos y epiclásticos (Branney y Kokelaar,
2002; Vallance, 2005). El proceso observado, en cámara lenta, proporciona nuevo material para investigar sobre los fenómenos de interacción entre partículas que llevan a la formación de estructuras de segregación. Estas estructuras han sido tradicionalmente relacionadas con procesos dinámicos que se desarrollan a lo largo del movimiento (Vallance, 2005). Sin embargo, la observación directa del proceso durante la formación y su cuantificación, sugieren que podrían ser otros los mecanismos involucrados, que posiblemente se encuentren relacionados con la segregación del material durante el deslizamiento. Los cambios de granulometría mostrados en la gráfica de la Figura 5 corresponden con seguridad al depósito de tres frentes secundarios distintos desarrollados en el mismo evento (Figura 3C).

\section{Conclusiones}

GRANFLOW-SIM, representa un importante desarrollo tecnológico y se trata del primer equipo de este tipo en 


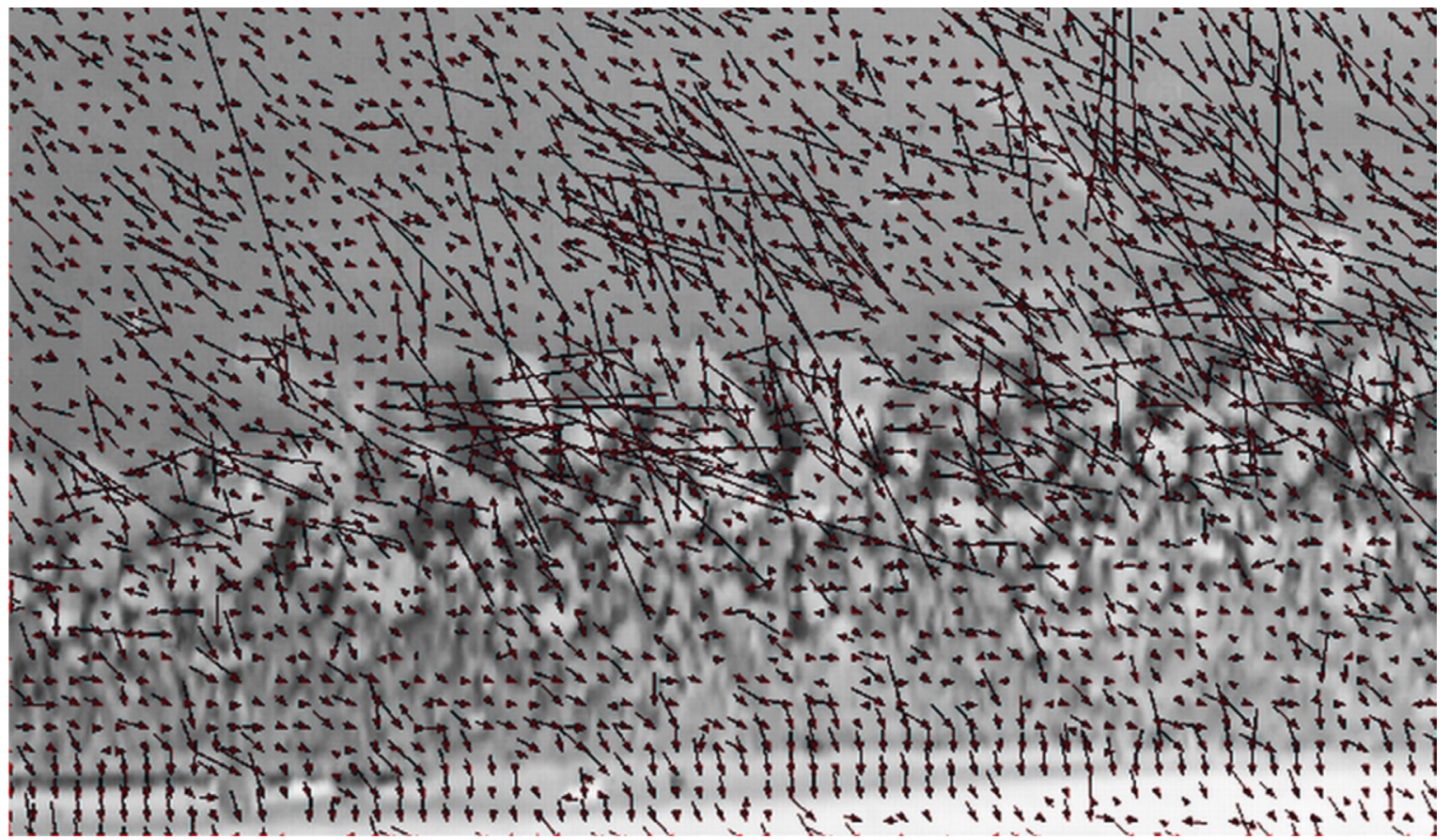

Figura 4. Ejemplo de análisis de velocimetría efectuado con el software desarrollado por Martínez-Ramirez y González (2006), en donde se observan los vectores de velocidad (dirección y magnitud) de las partículas.

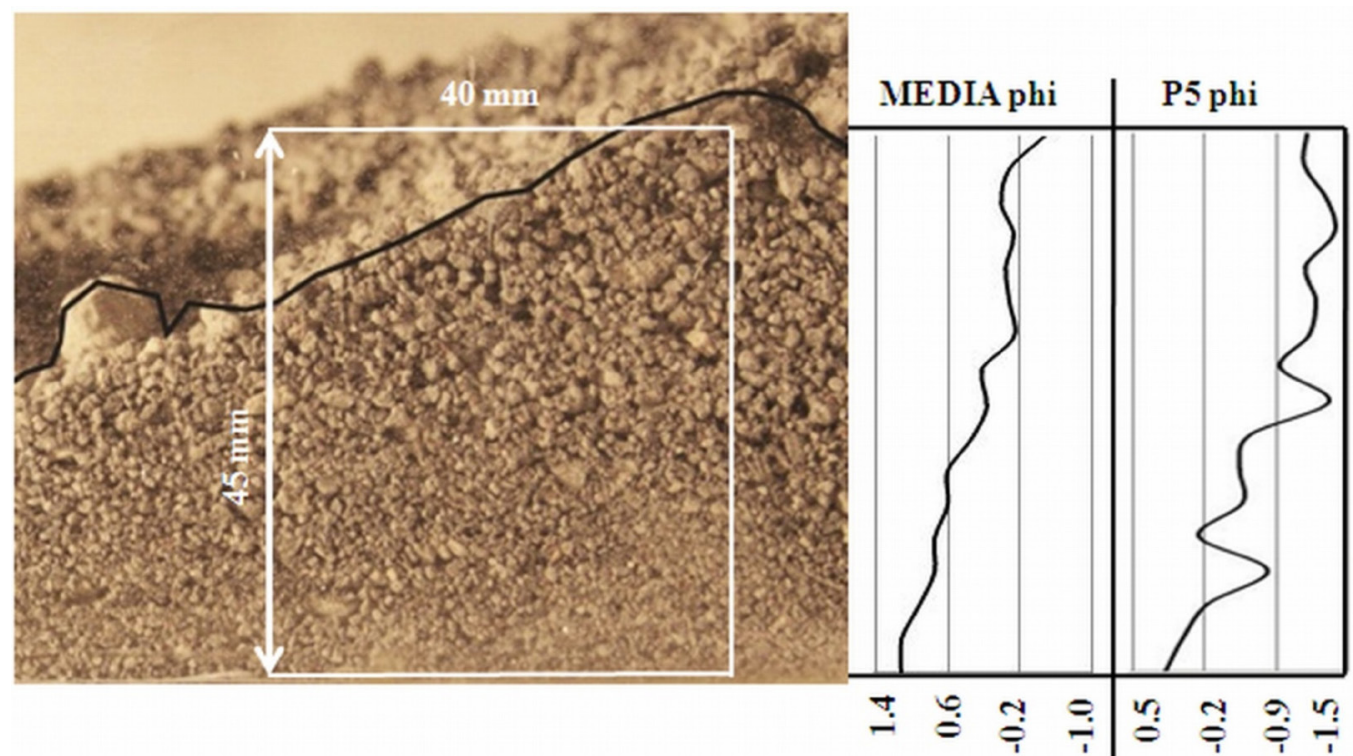

Figura 5. Perfil granulométrico vertical de un microdepósito analizado mediante el método del análisis de Rosiwal (Sarocchi et al., 2005). Los valores del tamaño promedio de los clastos (media) y el tamaño de los clastos mayores P5 ( $5^{\circ}$ percentil de la distribución) indican una clara gradación inversa.

México. Se encuentra a la vanguardia a nivel internacional como se ha podido comprobar mediante una extensiva investigación bibliográfica y con la presentación en encuentros internacionales especializados (Bartali et al., 2010).

La modularidad del conjunto y la presencia de una gran variedad de sensores, hacen de éste un instrumento versátil y flexible que le permite realizar una gama muy amplia de experimentos que no sólo tienen aplicación en el campo de la Geología, sino que pueden ser de interés para la industria.

Los resultados preliminares obtenidos, además de permitir la calibración de los sensores y de verificar el correcto funcionamiento de todo el sistema mecánico y electrónico, ya están proporcionando datos relevantes que podrán contribuir a mejorar la comprensión de los flujos granulares de tipo geológico. 


\section{Agradecimientos}

Se agradece a CONACyT (SEP-83301), PROMEP (UASLP-PTC-241), Fondos Concurrentes UASLP, por haber financiado el proyecto; al Instituto de Geología de la UASLP por permitirnos utilizar sus instalaciones; a Roberto Sulpizio y Lorenzo Borselli por los consejos durante el diseño del equipo y de los experimentos; a Anibal Montenegro por la asesoría brindada durante los experimentos; a Aldo A. Reyes Toral y Mireya Sarreón Villanueva por la invaluable ayuda durante la construcción; a Francisco Núñez Ruiz por la fabricación de las piezas mecánicas de precisión; y a Martha Elena Mondragón por haber fabricado los circuitos impresos del sistema electrónico.

\section{Referencias}

Bartali, R., Sarocchi, D., Nahmad-Molinary, Y., 2010, Modular apparatus for granular flow simulation (GRANFLOW-SIM): construction and applications (resumen), en Workshop on large scale experiments: Buffalo, New York, The State University of New York at Buffalo.

Boeolhouwers, J., Holness, S., Sumner, P., 2000, Geomorphological characteristics of small debris flows on Junior's Kop, Marion Island, maritime sub-Antarctic: Earth Surface Processes and Landforms, $25,341-352$.

Branney, M.J., Kokelaar, B.P., 2002, Pyroclastic density currents and the sedimentation of ignimbrites: Geological Society of London, Memoirs, 27, $152 \mathrm{p}$.

Cagnoli, B., Romano, G.P., 2010, Effect of grain size on mobility of dry granular flows of angular rock fragments: An experimental determination: Journal of Volcanology and Geothermal Research, 193, 18-24.

Capaccioni, B., Valentini, L., Rocchi, M.B.L., Nappi, G., Sarocchi, D., 1997, Image analysis and circular statistics for shape-fabric analysis: applications to lithified ignimbrites: Bulletin of Volcanology, 58, 501-514.

Capra, L., Macías, J.L., Scott, K.M., Abrams, M., Garduño-Monroy, V.H., 2002, Debris avalanches and debris flows transformed from collapses in the Trans-Mexican Volcanic Belt, Mexico - behavior, and implications for hazard assessment: Journal of Volcanology and Geothermal Research, 113, 81-110.

Carey, S.N., 1991, Transport and deposition of tephra by pyroclastic flows and surges, en Fisher, R.V., Smith, G.A. (eds.), Sedimentation in Volcanic Settings: SEPM (Society for Sedimentary Geology), Special Publication, 45, 39-57.

Cortés, A., Macías, J.L., Capra, L., Garduño-Monroy, V.H, 2010, Sector collapse of the SW flank of Volcán de Colima, Mexico: The 3600 yr BP La Lumbre-Los Ganchos debris avalanche and associated debris flows: Journal of Volcanology and Geothermal Research, 197,52 - 66.

Costa, J.E, 1988, Rheologic, geomorphic, and sedimentologic differentiation of water floods, hyperconcentrated flows, and debris flows, en Baker, V.R., Kochel, R.C., Patton, P.C. (eds.), Flood Geomorphology: New York, Wiley, 113-122.

Curry, R.R., 1966, Observation of alpine mudflows in the Tenmile Range, Central Colorado: Geological Society of America Bulletin, 77, 771-776.

DeGraff, J.V., 1994, The geomorphology of some debris flows in the southern Sierra Nevada, California: Geomorphology, 10, 231-252.
Drake, T.G., 1990, Structural features in granular flows: Journal of Geophysical Research, 95, 8681-8696.

Druitt, T.H., 1998, Pyroclastic density currents, en: Gilbert, J.S., Sparks, R.S.J. (eds.), The Physics of Explosive Volcanic Eruptions: Geological Society of London Special Publication, 145, 145-182.

Gorshkov, G.S, 1959, Gigantic eruption of the volcano Bezymianny: Bulletin Volcanologique, 20, 77-112.

Harris, S.A., Gustafson, C.A., 1993, Debris flow characteristics in an area of continuous permafrost, St. Elias Range, Yukon territory: Annals of Geomorphology, 37, 41-56.

Hoblitt, R.P., 1986, Observations of the eruptions of July 22 and August 7, 1980, at Mount St. Helens, Washington: U.S. Geological Survey, Professional Paper, 1335, 44 p.

Hsü, K.J., 1975, Catastrophic debris streams (Sturzstroms) generated by rockfalls: Geological Society of America Bulletin, 86, 129-140.

Hubert, J.F., Filipov, A.J., 1989, Debris-flow deposits in alluvial fans on the west flank of the White Mountains, Owens Valley, California, U.S.A.: Sedimentary Geology, 61, 177-205.

Huppert, H.E., Turner, J.S., Carey, S.N., Sparks, R.S.J., Hallworth, M.A., 1986, A laboratory simulation of pyroclastic flows down slopes: Journal of Volcanology and Geothermal Research, 30, 179-199.

Innes, J.L., 1983, Debris flows: Progress in Physical Geography, 7, 469501.

Ippolito, I., Annic, C., Lemaître, J., Oger, L., Bideau, D., 1995, Granular temperature: Experimental analysis: Physical Reviews, 52, 20722075.

Iverson, R.M., 1997, The physics of debris flows: Reviews of Geophysics, 35, 245-296.

Iverson, R.M., Schilling, S.P., Vallance, J.W., 1998, Objective delineation of lahar-inindation hazard zones: Geological Society of America Bulletin, 110, 972-984.

Jaeger, H.M., Nagel, S.R., Behringer, R.P., 1996, Granular solids, liquids, and gases: Reviews of Modern Physics, 68, 1259-1273.

Kadanoff, L.P., 1999, Built upon sand: Theoretical ideas inspired by granular flows: Reviews of Modern Physics, 71, 435-444.

Martínez-Ramírez, J.D., González, F.J., 2006, Velocímetro de partículas basado en imágenes digitales: San Luis Potosí, México, Universidad Autónoma de San Luis Potosí, Instituto de Investigación en Comunicación Óptica, tesis de maestría, 155 p.

Nieuwenhizen, M.E., van Steijn, H., 1990, Alpine debris flows and their sedimentary properties. A case study from the French Alps: Permafrost Periglacial Processes, 1, 111-128.

Palmer, B., Alloway, B.V., Neall, V.E., 1991, Volcanic debris avalanche deposits in NewZealand - lithofacies organization in unconfined, wetavalanche flows, en Fisher, R.V., Smith, G.A. (eds.), Sedimentation in Volcanic Settings: SEPM (Society for Sedimentary Geology), Special Publication, 45, 89-98.

Pareschi, M.T., Favalli, M., Giannini, F., Sulpizio, R., Zanchetta, G., Santacroce, R., 2000, May 5, 1998, debris flows in circum-Vesuvian areas (southern Italy): Insights for hazard assessment: Geology, 28, 639-642.

Pierson, T.C., 1980, Erosion and deposition by debris flows at Mt. Thomas, North Canterbury, New Zealand: Earth Surface Processes and Landforms, 5, 227-247

Rickenmann, D., Zimmermann, M., 1993, The 1987 debris flows in Switzerland: documentation and analysis: Geomorphology, 8 , 175-189.

Roche, O., Montserrat, S., Niño, Y., Tamburrino, A., 2010, Pore fluid pressure and internal kinematics of gravitational laboratory air-particle flows: Insights into the emplacement dynamics of pyroclastic flows: Journal of Geophysical Research, 115, B09206, doi: 1029/2009JB007133.

Rodolfo, K.S., 2000, The hazards from lahars and jökulhlaups, en Sigurdsson, H., Houghton, B.F., McNutt, S.R., Rymer, H., Stix, J. (eds.), Encyclopedia of Volcanoes: Londres, Academic Press, 973-995.

Sarocchi, D., Borselli, L., Macias, J.L., 2005, Construcción de perfiles granulométricos de depósitos piroclasticos por métodos ópticos: Revista Mexicana de Ciencias Geologicas, 22, 371-382. 
Sarocchi D., 2007, Estudio sedimentológico del depósito de flujo de bloques y ceniza del 17 de julio de 1999 en el volcán de Colima: México, Universidad Nacional Autónoma de México, Monografiás del Instituto de Geofísica, 11, 120 p.

Sato, H., Fujii, T., Nakada, S., 1992, Crumbling of dacite dome lava and generation of pyroclastic flows at Unzen Volcano: Nature, 360, 664-666.

Saucedo, R., 1997, Reconstrucción de la Erupción de 1913 del Volcán de Colima: México D.F., Universidad Nacional Autónoma de México, Instituto de Geofísica, tesis de maestría, $185 \mathrm{p}$.

Sharp, R.P., Nobles, L.H., 1953, Mudflow of 1941 at Wrightwood, southern California: Geological Society of America Bulletin, 64, 547-560.

Siebert, L., 1984, Large volcanic debris avalanches: Characteristics of source areas, deposits, and associated eruptions: Journal of Volcanology and Geothermal Research, 22, 163-197.

Sulpizio, R., Dellino, P., 2008, Sedimentology, depositional mechanisms and pulsating behaviour of pyroclastic density currents, en Gottsman, J., Martí, J. (eds.), Caldera Volcanism: Analysis, Modelling and Response: Amsterdam, Elsevier, 57-96.

Sulpizio R., Capra, L., Sarocchi, D., Saucedo, R., Gavilnes-Ruiz, J.C., Varley, N.R., 2010, Predicting the block-and-ash flow inundation areas at Volcán de Colima (Colima, Mézxico) based on the present day (February 2010) status: Journal of Volcanology and Geothermal Research, 193, 49-66.

Vallance, J.W., 2000, Lahars, en Sigurdsson, H. (ed.), Encyclopedia of Volcanoes: Londres, Academic Press, 601-616.
Vallance, J.W. 2005 Volcanic debris flows, en Jakob, M., Hungr, O. (eds.), Debris-flow hazards and related phenomena: Alemania, Springer Berlin Heidelberg, Praxis, 247-271.

Van Steijn, H., de Ruig, J., Hoozemans, F., 1988, Morphological and mechanical aspects of debris flows in parts of the French Alps: Zeitschrift für Geomorphologie, 32, 143-161.

Voight, B., Glicken, H., Janda, R.J., Douglass, P.M., 1981, Catastrophic rockslide avalanche of May 18, en Lipman, P.W., Mullineaux, D.R. (eds.), The 1980 eruptions of Mount St. Helens: Washington, U. S. Geological Survey, Professional Paper 1250, 347-377.

Yamasato, H., 1997, Quantitative analysis of pyroclastic flows using infrasonic and seismic data at Unzen Volcano, Japan: Journal of Physics of the Earth, 45, 397-416.

Manuscrito recibido: Diciembre 18, 2010

Manuscrito corregido recibido: Enero 15, 2011.

Manuscrito aceptado: Enero 19, 2011. 